SHORT COMMUNICATION

\title{
High Frequency Dynamic Ultrasound Scattering from Microsphere Suspensions
}

\author{
By Mariko KoHyama, Tomohisa NORISUYe, ${ }^{*}$ and Qui Tran-CONG-MIYATA
}

KEY WORDS: Ultrasound / Scattering / Correlation Function / Microsphere / Dynamics /

Dynamic light scattering (DLS) now becomes one of the most popular tools to investigate the structure and dynamics of polymer solutions, suspensions and gels. This method utilizes the so-called time correlation functions, calculated from temporal scattering intensity fluctuations resulting from the particle motion. ${ }^{1}$ The typically observable length scales range from several to hundreds of nanometers. DLS may be utilized to monitor even larger structures up to several microns. However DLS requires great knowledge about the structure factor and internal modes.

As the length scale of a given structure approaches micrometer scale, permeability of visible light becomes poor and multiple scattering also increases. Thus novel techniques that enable structure analysis for opaque objects would be strongly demanded to overcome this problem. Recently, Page and co-workers proposed a dynamic sound scattering (DSS) technique to investigate a complex flow by using glass beads ranging from millimeters to sub-millimeters. ${ }^{2,3}$ In contrast to the conventional Doppler ultrasound techniques, ${ }^{4}$ DSS can monitor the more complex dynamics by analyzing time evolution of speckle intensity in analogy to the conventional DLS. In order to utilize DSS for monitoring micrometer or even smaller size, we have explored the DSS using longitudinal wave with the frequency $20 \mathrm{MHz}$. Although the attenuation becomes more serious, the corresponding wavelength $(\lambda=75 \mu \mathrm{m}$ in water) is ten times shorter than the previous experiments, enabling us to probe the dynamics of polymer dispersions in a range of several to several tens of micrometers.

\section{EXPERIMENTAL}

\section{Samples}

Standard Latex microsphere suspensions were purchased from Duke Scientific Corporation. The particles are redispersed in an aqueous solution to obtain suspension with desired concentrations in the range $0.5<$ $c<3 \mathrm{wt} \%$. The particle size, coefficient of variation (CV) and density are summarized in Table I. SEM micrographs (Hitachi S-3000N) were also taken to verify the particle size and their distribution. Other Latex particles with the average diameter 3.5 and $5.5 \mu \mathrm{m}$ were supplied by courtesy of Sekisui Chemical Co. Ltd. All the DSS experiments were performed at $20.4 \pm 0.05^{\circ} \mathrm{C}$.

\section{Apparatus}

Negative impulse emitted from a pulser/receiver (Olympus, model $5800 \mathrm{PR}$ ) was transferred to a $20 \mathrm{MHz}$-longitudinal plane wave transducer (Olympus, model V317) immersed in a water bath to generate broadband ultrasound pulse. The reflected or scattered ultrasound wave was received by the same transducer. The obtained signals were then amplified by the receiver, followed by successive recording with a 14 bit high-speed digitizer (Compuscope CS14200) at the sampling rate $200 \mathrm{Ms} / \mathrm{s}$.

\section{RESULTS AND DISCUSSION}

So far, optical techniques have utilized variation of pulse "intensities" as illustrated in Figure 1(a) because the electric field of the scattered light is too fast to be digitized. On the other hand, in the case of DSS, propagation of the sound wave including ballistic and scattering waves, is slow enough to be resolved in the pulse propagation time by the latest digitizer with an appropriate sampling rate. ${ }^{5}$ This enables us not only to determine phase of the scattering wave but also to extract the desired information at a specific scattering position or scattering path. Figure 1(b) shows a snap shot of propagating waveform of the $45 \mu \mathrm{m}$ polystyrene beads. As illustrated in Figure 1(c), the waveform includes four reflected components at the front and rear interfaces for two plastic walls. Ultrasound scattering should be observed between two-cell walls if the object scale is comparable with the source wavelength.

Such a ultrasound pulse, indicated by the scattering region in Figure 1(b), was repetitively captured. The evolution profile was further processed by a PC off-line analysis to evaluate the time-field correlation function.

Time-field correlation functions are defined as,

$$
g^{(1)}(q, \tau)=\langle\exp [-i q \cdot \Delta r(\tau)]\rangle_{T}
$$

where $q\left(=0.169 \mu \mathrm{m}^{-1}\right)$ is the magnitude of the scattering vector, $\Delta r$ is the displacement of the microsphere during the time interval $\tau$. The bracket indicates the evolution time average. $\Delta r$ may be rewritten as a sum of the average displacement and the deviation from the average,

$$
\Delta r(\tau)=\langle\Delta r\rangle+\delta r(\tau)
$$

By substituting eq 2 into eq 1 , one obtains

$$
g^{(1)}(q, \tau) \simeq \cos (q\langle\Delta r(\tau)\rangle) \times \exp \left(-\frac{1}{2} q^{2}\left\langle\delta r^{2}(\tau)\right\rangle\right)
$$

In the case of aqueous suspensions of microsphere, sedimentation can dominate the dynamics. However, the detectable information depends on the direction of the wave vector with respect to the sedimentation direction. If the scattering vector is parallel (so is the beam angle) to the sedimentation direction, the average sedimentation velocity along this direction, $\left\langle V_{z}\right\rangle$, and its variance, $\left\langle\delta V_{z}{ }^{2}\right\rangle$, can be detected. On the other hand, when the incident beam is normal to the sedimentation direction, the time correlation functions contain only the velocity variance, $\left\langle\delta V_{y}{ }^{2}\right\rangle$, since the wave vector does not coincide with the sedimentation direction.

Figure 2 demonstrates the time correlation functions (a) $g_{z}^{(1)}(\tau)$ and (b) $g_{y}{ }^{(1)}(\tau)$ for an $11 \mu \mathrm{m}$ microsphere. The correlation functions obtained from the different propagation times were exhibited all together in the figure. The relaxation time, $\left(=2^{1 / 2} q^{-1}\left\langle\delta V^{2}\right\rangle^{-1 / 2}\right)$ became faster as the propagation time increased because the multiply scattered waves became uncorrelated

Department of Macromolecular Science and Engineering, Graduate School of Science \& Technology, Kyoto Institute of Technology, Matsugasaki, Sakyo-ku, Kyoto 606-8585, Japan

*To whom correspondence should be addressed (Tel: +81-75-724-7853, Fax: +81-75-724-7853, E-mail: nori@ kit.jp). 
Table I. Characteristics of the particles used in this study

\begin{tabular}{ccccc}
\hline$d(\mu \mathrm{m})$ & $\mathrm{CV}$ & $\rho\left(\mathrm{g} / \mathrm{cm}^{3}\right)$ & $\left\langle V_{z}\right\rangle(\mathrm{mm} / \mathrm{s})$ & $d_{\text {DSs }}(\mu \mathrm{m})$ \\
\hline 45 & 0.15 & 1.05 & $5.11 \times 10^{-2}$ & 42.50 \\
\hline 11 & 0.18 & 1.05 & $3.40 \times 10^{-3}$ & 10.96 \\
\hline 5.5 & 0.05 & 1.18 & $3.12 \times 10^{-3}$ & 5.61 \\
\hline 3.5 & 0.07 & 1.18 & $1.28 \times 10^{-3}$ & 3.59 \\
\hline
\end{tabular}

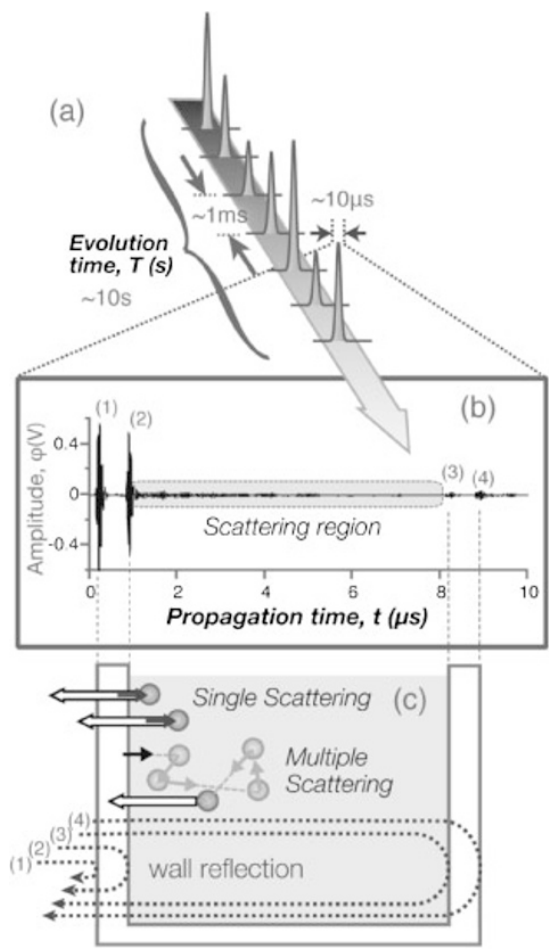

Figure 1. (a) Schematic representation of the time evolution of the pulse intensity (or magnitude); (b) an example for $20 \mathrm{MHz}$ ultrasound pulse and (c) the interpretation for reflecting and scattering waves.

for the longer path length. ${ }^{6}$ As shown in Figure 2(a), the correlation functions involved some oscillations during the relaxation. This is the characteristic behavior accompanying sedimentation whose average displacement is non-zero as described by eq 3 . As demonstrated in the figure, curve fitting using eq 3 was satisfactory particularly for the longer relaxation curve. On the other hand, the $y$-component of the average velocity, $\left\langle V_{y}\right\rangle$ was zero, resulting in the monotonic relaxation without cosine oscillation. After evaluation of $\left\langle V_{z}\right\rangle$ at the field time for a single scattering event, $\left\langle V_{z}\right\rangle$ was further extrapolated to the infinite dilute concentration to obtain the interaction-free mean velocity of the particles.

Table I summarizes the density and average velocity for the various microspheres studied here. $\left\langle V_{z}\right\rangle$ was further transformed into the particle diameter by using the hydrodynamic formula,

$$
d_{\mathrm{DSS}}=\sqrt{18 \eta_{0}\left\langle V_{z}\right\rangle /\left(\rho-\rho_{0}\right) g}
$$

where $\eta_{0}$ and $\rho_{0}$ are the viscosity and density for solvent respectively. While most of the data exhibited good agreement between the provided and the calculated particle diameters, the result for the $45 \mu \mathrm{m}$ beads was underestimated in spite of the high $\mathrm{S} / \mathrm{N}$ ratio. This is probably due to the probing range determined by the reduced wave vector $Q=q d / 2$. Although
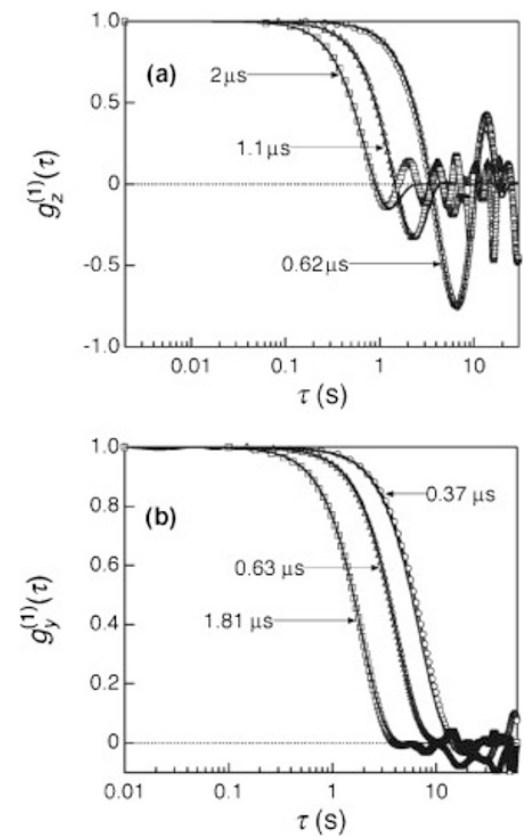

Figure 2. The time-field correlation functions of the $11 \mu \mathrm{m}$ polystyrene beads obtained for (a), $z$ - and (b), $y$-components of sedimentation dynamics.

we have shown a preliminary high frequency DSS results in this study, further information can be obtained if the scattering angle dependence is explored under different geometries. As the particle size becomes smaller, the diffusion behavior is also important to be discussed. It will be addressed in the forthcoming paper.

Acknowledgment. This work is supported by Grant-in-Aid, No 18750190 and Grant-in-Aid for Scientific Research on Priority Area, "Soft Matter Physics" (No. 463/19031018) from the Ministry of Education, Science, Sports, Culture, and Technology. The authors are acknowledged to Drs. J. H. Page and A. Strybulevych, Mr. K. Hildebrand for valuable advises for this study.

Received: January 1, 2008

Accepted: February 3, 2008

Published: March 19, 2008

\section{REFERENCES}

1. B. J. Berne and R. Pecora, "Dynamic Light Scattering with Applications to Chemistry, Biology and Physics," Dover Publications, Inc., Mineola, N. Y., 2000.

2. M. L. Cowan, J. H. Page, and D. A. Weitz, Phys. Rev. Lett., 85, 453 (2000).

3. A. Strybulevych and J. H. Page, Microgravity Sci. Technol., 16, 249 (2005).

4. D. H. Evans, "Doppler Ultrasound: Physics, Instrumental, and Clinical Applications," Wiley, New York, 2000.

5. T. Norisuye, A. Strybulevych, and M. Scanlon, Macromol. Symp., 242, 208 (2006).

6. M. L. Cowan, I. P. Jones, J. H. Page, and D. A. Weitz, Phys. Rev. E, 65, 0666605 (2002). 